\title{
SELETIVIDADE DE INSETICIDAS A Podisus rostralis (Stal) (HETEROPTERA: PENTATOMIDAE) PREDADOR DE LAGARTAS DESFOLHADORAS DE EUCALIPTO ${ }^{1}$
}

\author{
Alfredo Henrique Rocha Gonring², Marcelo Coutinho Picanço², Germano Leão Demolin Leite ${ }^{4}$, Fábio Akiyoshi \\ Suinaga ${ }^{5}$ e José Cola Zanuncio ${ }^{2}$
}

\begin{abstract}
RESUMO - O objetivo deste trabalho foi estudar a seletividade fisiológica dos inseticidas deltametrina, fenitrotiom, malatiom e permetrina em favor de ninfas ( $3^{\circ}$ e 5o instares) e adultos (fêmeas e machos) do predador Podisus rostralis, empregando-se 50 (subconcentração) e 100\% da concentração usada no controle de lagartas desfolhadoras de eucalipto. Deltametrina foi seletivo em favor de ninfas de 3e 5o instares e adultos (1,94, 0,00 e 7,87\% de mortalidade, respectivamente) do predador. Permetrina foi seletivo em favor de ninfas de 5o instar $(3,55 \%$ de mortalidade) e medianamente seletivo em favor de ninfas de 3 o instar e adultos $(28,22$ e 32,08\% de mortalidade, respectivamente) do predador. Malatiom foi medianamente seletivo em favor de ninfas de 5o instar (49,38\% de mortalidade) e não-seletivo em favor de ninfas 3 - instar e adultos (95,34 e 88,99\% de mortalidade, respectivamente) do predador. Fenitrotiom não foi seletivo em favor de nenhum dos instares do predador (100\% de mortalidade). A alta toxicidade do fenitrotiom a $P$. rostralis não foi reduzida quando o predador foi exposto a $50 \%$ da concentração recomendada deste inseticida. O impacto do malatiom (a ninfas de 3 instar e adultos) e permetrina (a fêmeas) sobre $P$. rostralis diminuiu quando estas fases do predador foram expostas à metade das concentrações desses inseticidas. O impacto do deltametrina não foi afetado por sua concentração. Ninfas de 3o instar e adultos foram menos tolerantes do que ninfas de 5 o instar ao malatiom e permetrina. As fêmeas de $P$. rostralis foram mais tolerantes que os machos à concentração do malatiom e à subconcentração de permetrina.
\end{abstract}

Palavras-chave: Percevejo predador, deltametrina, fenitrotiom, malatiom e permetrina.

\section{SELECTIVITY OF INSECTICIDES TO Podisus rostralis (Stal) (HETEROPTERA: PENTATOMIDAE) PREDATOR OF EUCALYPTUS-DEFOLIATING CATERPILLARS}

\begin{abstract}
The objective of this work was to study the physiological selectivity of deltamethrin, fenitrothion, malathion, and permethrin to nymphs (third and fifth instars) and adults (females and males) of the predator Podisus rostralis. The insecticides were used in concentrations corresponding to 50 and $100 \%$ of the concentration used for control of eucalyptus-defoliating caterpillars. Deltamethrin was selective to third and fifth instar nymphs and adults of the predator (1.94, 0.00 and 7.87\% of mortality, respectively). Permethrin was selective in favor of fifth instar nymphs (3.55\% of mortality), and showed median selectivity in favor of third instar nymphs and adults of the predator (28.22 and 32.08\% of mortality). Malathion was medially selective to fifth instar nymphs (49.38\% of mortality), but was not selective to fifth instar nymphs and adults of the predator (95.34 and $88.99 \%$ of mortality). Fenitrothion was not selective to any instar of $\boldsymbol{P}$. rostralis (100\% of mortality). The high toxicity of fenitrothion to this predator did not decrease when the lowest concentration was used, but the impact of malathion (to third instar nymphs and adults) and permethrin (to females) of $\boldsymbol{P}$. rostralis was reduced when these instars were exposed to half of the concentrations of these insecticides. The impact of deltamethrin was not affected by the concentration. Finally, third instar nymphs and adults were less tolerant to malathion and permethrin than fifth instar nymphs of the predator; females of $\boldsymbol{P}$. rostralis were more tolerant than males to the recommended concentration of malathion and to half permethrin.
\end{abstract}

Key words: $\quad$ Predator bug, deltamethrin, fenitrothion, malathion, permethrin.

1 Recebido para publicação em 18.5.2000.

Aceito para publicação em 19.2.2003.

2 Dep. de Biologia Animal da Universidade Federal de Viçosa - UFV, 36571-000 Viçosa-MG, <picanco@mail.ufv.br>. ${ }^{4}$ Dep. de Fitotecnia da Universidade Federal de Minas Gerais - UFMG, 39404-006 Montes Claros-MG. ${ }^{5}$ Embrapa - Algodão, 58107-720 Campina Grande-PB. 


\section{INTRODUÇÃO}

As lagartas desfolhadoras (Lepidoptera) estão entre as principais pragas de eucalipto no Brasil. Uma das principais táticas de manejo integrado dessas pragas é a utilização de inimigos naturais, além do controle químico (Zanuncio et al., 1992a, e 1993b; Suinaga et al., 1996). Os percevejos pertencentes à família Pentatomidae são os principais predadores de lagartas desfolhadoras de eucalipto, destacando-se o gênero Podisus. Entre as espécies mais importantes desse gênero está Podisus rostralis (Stal) (Heteroptera: Pentatomidae) (Zanuncio et al., 1993b).

Entretanto, para compatibilização dos controles químico e biológico em programas de Manejo Integrado de Pragas, é de fundamental importância o uso de inseticidas eficientes contra as pragas e seletivos em favor dos inimigos naturais, para possibilitar a preservação destes agentes do controle biológico (Gonring et al., 1999). A seletividade de inseticidas pode ser classificada em ecológica e fisiológica. A seletividade ecológica é obtida pela adoção de técnicas de aplicação que minimizem a exposição do inimigo natural ao inseticida. Já a seletividade fisiológica é obtida pelo emprego de inseticidas seletivos, ou seja, de compostos que são mais tóxicos às pragas do que aos inimigos naturais, devido a variações na sensibilidade destes organismos ao praguicida (Pedigo, 1988). Neste trabalho o termo seletividade refere-se à seletividade fisiológica.

No estudo da seletividade de inseticidas, o uso das concentrações recomendadas para o controle das pragas possibilita a avaliação do impacto destes produtos no momento de sua aplicação. Já o uso de subconcentrações, por exemplo $50 \%$ da recomendação, permite o estudo do impacto dos inseticidas quando estes forem decompostos à metade de suas concentrações originais (Guedes et al., 1992; Suinaga et al., 1996). Essa informação permite determinar o tempo necessário após a aplicação do inseticida para realização de liberações do inimigo natural no campo ou mesmo conhecer o impacto de resíduos do inseticida sobre populações imigrantes do inimigo natural.

Entre os principais inseticidas utilizados no controle de lagartas desfolhadoras de eucalipto estão: deltametrina, fenitrotiom, malatiom e permetrina (Zanuncio et al., 1993a; Suinaga et al., 1996). Entretanto, existem poucos trabalhos sobre seletividade de inseticidas envolvendo inimigos naturais de lagartas desfolhadoras de eucalipto (Guedes et al., 1992; Suinaga et al., 1996). Assim, com o intuito de gerar subsídios a programas de Manejo Integrado de Pragas de eucalipto, neste trabalho objetivou-se estudar a seletividade fisiológica dos inseticidas deltametrina, fenitrotiom, malatiom e permetrina, usados no controle de lagartas desfolhadoras, em favor de ninfas de $3^{\circ}$ e $5^{\circ}$ instares, fêmeas e machos do percevejo predador $P$. rostralis, quando expostos a 50 e $100 \%$ das concentrações destes inseticidas, usadas no controle destes insetos-praga.

\section{MATERIAL E MÉTODOS}

Esta pesquisa foi conduzida de abril a maio de 1996, no Laboratório de Manejo Integrado de Pragas da Universidade Federal de Viçosa (UFV), Viçosa-MG. Nos bioensaios foram utilizados insetos obtidos da criação massal de Podisus rostralis do Laboratório de Entomologia Florestal (UFV), conforme técnica proposta por Zanuncio et al. (1992a).

Os inseticidas foram aplicados em concentrações que correspondem a 50 (subconcentração) e $100 \%$ das concentrações recomendadas para o controle de lagartas desfolhadoras de eucalipto. Os inseticidas e as concentrações (em mg de i.a./ml de calda) empregados foram: os organofosforados fenitrotiom $500 \mathrm{CE}(0,5$ e 1,0) e malatiom $500 \mathrm{CE}$ (2 e 4) e os piretróides deltametrina $25 \mathrm{CE}(0,005$ e 0,01$)$ e permetrina $500 \mathrm{CE}(0,05$ e 0,1$)$. Em todos os tratamentos foi adicionado o espalhante adesivo N-Dodecil benzeno sulfonato de sódio $320 \mathrm{CE}$ na concentração de $30 \mathrm{ml} / 1001$ de calda (ANDREI, 1996). $\mathrm{Na}$ testemunha utilizou-se somente água mais espalhante adesivo.

O delineamento experimental foi inteiramente casualizado em arranjo fatorial de 4 x 4 x 2 (inseticidas $\mathrm{x}$ instares de desenvolvimento $\mathrm{x}$ concentrações dos inseticidas) com quatro repetições, além da testemunha. Adultos sexados e ninfas de 3 ㅇ e 5o instares foram expostos aos inseticidas, por meio de folhas de Eucalyptus grandis W. Hill ex Maiden. Para tanto, as folhas foram imersas em calda inseticida por cinco segundos, conforme os tratamentos experimentais, deixadas para secar por 2 horas. Em seguida, três folhas foram cortadas e alojadas por placas de Petri, de $9 \mathrm{~cm}$ de diâmetro por $2 \mathrm{~cm}$ de altura, de forma a cobrir todo o fundo da placa. Em cada unidade experimental, liberaram-se dez percevejos (ninfas de $3^{\circ}$ instar, ninfas de $5^{\circ}$ instar, fêmeas ou machos). As placas foram cobertas com organza, que foi fixada por meio de elástico. Posteriormente, as placas foram acondicionadas em estufa 
incubadora, à temperatura de $25 \pm 0,5^{\circ} \mathrm{C}$ e $75 \pm 5 \%$ de umidade relativa. Vinte e quatro horas depois foi realizada a avaliação da mortalidade dos insetos. Os resultados foram corrigidos em relação à mortalidade ocorrida na testemunha, utilizando a fórmula de Abbott (1925).

Os resultados de mortalidade de $P$. rostralis foram submetidos à análise de variância (ANOVA) e, posteriormente, as médias foram comparadas pelo teste de Scott-Knott a $\mathrm{P}<0,05$ (Scott \& Knott, 1974), utilizando o programa Sistema de Análise Estatística e Genética (SAEG) (Euclides, 1983).

O critério de avaliação da seletividade foi com base no conceito de seletividade fisiológica, no qual o inseticida é classificado como seletivo se causar mortalidade na população do inimigo natural muito menor do que na população da praga. Ele é considerado como não-seletivo se causar mortalidade na população do inimigo natural maior ou igual à ocorrida na população da praga; se o inseticida está em situação intermediária a estes dois extremos, ele é considerado como medianamente seletivo (Pedigo, 1988; Yu, 1988; Guedes et al., 1992; Faleiro et al., 1995). Segundo Zanuncio et al. (1992b, 1993b) e Suinaga et al. (1996), os inseticidas utilizados neste bioensaio ocasionam mortalidade de cerca de $90 \%$ às lagartas desfolhadoras de eucalipto. Assim, os inseticidas foram considerados como seletivos se ocasionaram mortalidade estatisticamente semelhante a $0 \%$; nãoseletivos se ocasionaram mortalidade estatisticamente maior ou igual a 90\%; e medianamente seletivos se ocasionaram mortalidade estatisticamente menor que $90 \%$ e maior que $0 \%$ pelo teste de Scott-Knott, a $\mathrm{P}<0,05$.

\section{RESULTADOS E DISCUSSÃO}

Nas concentrações recomendadas para o controle de lagartas desfolhadoras de eucalipto, o fenitrotiom não apresentou seletividade em favor do predador Podisus rostralis, ocasionando mortalidade de $100 \%$ aos três instares estudados. $\mathrm{O}$ malatiom não foi seletivo em favor de ninfas de $3^{\circ}$ instar e adultos, e medianamente seletivo em favor de ninfas de $5 \circ$ instar. O permetrina foi medianamente seletivo em favor de ninfas de $3^{\circ}$ instar e adultos e seletivo em favor de ninfas de 5 ํ instar. O deltametrina foi seletivo em favor de todos os instares do predador (Quadro 1).

Há relatos na literatura que indicam a seletividade de inseticidas piretróides, como deltametrina e permetrina, em favor de inimigos naturais de distintos grupos taxonômicos, incluindo Heteroptera: Pentatomidae; Dermaptera: Forficulidae; Neuroptera: Chrysopidae; Hymemoptera: Braconidae, Ichneumonidae, Trichogrammatidae e Vespidae (Rajakulendran \& Plapp Jr., 1982; Powell et al., 1986; Singh \& Guardial, 1986; Yu, 1988; Guedes et al., 1992; Batalha et al., 1995; Faleiro et al., 1995; Suinaga et al., 1996; Picanço et al., 1997; Picanço et al., 1998; Gonring et al., 1999).

A seletividade dos piretróides pode ser devido à sua menor taxa de penetração no integumento (Guedes et al., 1992) ou à maior metabolização ou alterações no sítio de ação destes inseticidas nos inimigos naturais do que nas pragas (Yu, 1988; Suinaga et al., 1996).

As ninfas de 5o instar foram mais tolerantes às concentrações recomendadas para controle de lagartas desfolhadoras de eucalipto dos inseticidas malatiom e permetrina que as ninfas de $3^{\circ}$ instar e os adultos (Quadro 1). Resultados semelhantes foram obtidos para P. nigrispinus, por Batalha et al. (1995) e Suinaga et al. (1996), e para S. cincticeps, por Batalha et al. (1995). A maior suscetibilidade das ninfas de 3ํㅡㄴ instar e dos adultos a tais concentrações desses inseticidas pode estar relacionada à menor capacidade de desintoxicação nestas fases (Hollingworth, 1976; Picanço et al., 1997).

As fêmeas de $P$. rostralis foram mais tolerantes que os machos à concentração do malatiom usada no controle de lagartas de eucalipto. Elas foram mais tolerantes que os machos a $50 \%$ da concentração de permetrina usada no controle de lagartas de eucalipto (Quadro 2). Fato semelhante foi observado por Suinaga et al. (1996) para P. nigrispinus, usando subconcentração de malatiom. A menor suscetibilidade de fêmeas a estes inseticidas, nestas concentrações, pode ser atribuída à maior quantidade de gordura nas fêmeas do que nos machos (Legaspi et al., 1996), visto que o tecido adiposo funciona como "sequiestrador" do ingrediente ativo, tornando-o inerte no organismo (Winteringham, 1969). Além disto, Picanço et al. (1997) relataram que a maior tolerância das fêmeas aos inseticidas pode ser atribuída à sua maior atividade desintoxificativa, devendo ser ressaltado que esta maior atividade metabólica estaria relacionada à maior reserva energética das fêmeas do que dos machos, por elas terem necessidade de maiores gastos energéticos na fase reprodutiva.

Assim, pode-se concluir que, em programas de controle biológico com liberação desse predador em florestas com uso de permetrina e, ou, malatiom, deve-se 
liberar ninfas de 5ำ instar, por ele o estádio mais tolerante a estes inseticidas. Já se o programa de controle biológico é realizado com liberação de adultos, devem ser liberadas fêmeas previamente copuladas em laboratório ou um maior número de machos que fêmeas para garantir a sua fecundação, uma vez que os machos são menos tolerantes a estes inseticidas.
O alto índice de mortalidade causado pelo fenitrotiom na concentração recomendada manteve-se quando foi utilizada metade de sua concentração para ninfas de $3^{\circ}$ e 5 instares e adultos de ambos os sexos (Quadros 1 e 2). Infere-se, portanto, que quando o fenitrotiom é usado no controle de lagartas em eucalipto ele apresenta alto impacto a tais instares desse percevejo

Quadro 1 - Mortalidade (média \pm erro-padrão) de adultos e ninfas de terceiro e quinto instares de Podisus rostralis causada por duas concentrações de quatro inseticidas utilizados no controle de lagartas-desfolhadoras de eucalipto

Table 1 - Mortality (mean \pm stand error) of adults and third and fifth instar nymphs of Podisus rostralis caused by two concentrations of four insecticides used to control eucalyptus defoliating caterpillars

\begin{tabular}{|l|c|c|c|c|c|c|}
\hline \multirow{3}{*}{ Inseticida } & \multicolumn{6}{|c|}{ Fase* } \\
\cline { 2 - 8 } & Ninfa III & Ninfa V & Adulta & Ninfa III & Ninfa V & Adulta \\
\cline { 2 - 8 } & \multicolumn{3}{|c|}{ (Concentração recomendada) } & \multicolumn{4}{c|}{ (50\% da concentração) } \\
\hline Fenitrotiom & $100,00 \pm 0,00 \mathrm{aA} \alpha$ & $100,00 \pm 0,00 \mathrm{aA} \alpha$ & $100,00 \pm 0,00 \mathrm{aA} \alpha$ & $100,00 \pm 0,00 \mathrm{aA} \alpha$ & $100,00 \pm 0,00 \mathrm{aA} \alpha$ & $100,00 \pm 0,00 \mathrm{aA} \alpha$ \\
Malatiom & $95,34 \pm 2,64 \mathrm{aA} \alpha$ & $49,38 \pm 13,75 \mathrm{bB} \alpha$ & $88,99 \pm 6,43 \mathrm{aA} \alpha$ & $58,15 \pm 10,84 \mathrm{aB} \beta$ & $61,77 \pm 13,29 \mathrm{aB} \alpha$ & $65,95 \pm 6,33 \mathrm{aB} \beta$ \\
Permetrina & $28,22 \pm 17,86 \mathrm{aB} \alpha$ & $3,55 \pm 2,06 \mathrm{bC} \alpha$ & $32,08 \pm 3,19 \mathrm{aB} \alpha$ & $11,23 \pm 7,50 \mathrm{aC} \alpha$ & $3,78 \pm 2,19 \mathrm{aC} \alpha$ & $14,23 \pm 9,39 \mathrm{aC} \beta$ \\
Deltametrina & $1,94 \pm 1,94 \mathrm{aC} \alpha$ & $0,00 \pm 0,00 \mathrm{aC} \alpha$ & $7,87 \pm 4,66 \mathrm{aC} \alpha$ & $0,00 \pm 0,00 \mathrm{aC} \alpha$ & $3,55 \pm 2,06 \mathrm{aC} \alpha$ & $0,84 \pm 0,84 \mathrm{aC} \alpha$ \\
\hline
\end{tabular}

* As médias seguidas pela mesma letra maiúscula na coluna (para comparação entre inseticidas), minúscula na linha (para comparação das diferenças de tolerância das fases do inseto a uma mesma concentração de cada inseticida) ou do alfabeto grego na linha (para comparação entre concentração recomendada e $50 \%$ da concentração de cada inseticida a determinada fase do inseto) não diferem entre si, pelo teste de Scott - Knott a $\mathrm{P}<0,05$.

* Means followed by same capital letter in a column (comparison between insecticides), lower case letter in row (for comparison of the differences of tolerance of the phases of insect to a similar concentration of insecticide), or of a Greek letter in a row (for comparison of the recommended concentration and 50\% of concentration of each insecticide to a particular phase of insect) are not significantly different by the Scott-Knott test at $p<0.05$.

Quadro 2 - Mortalidade (média \pm erro padrão) de fêmeas e machos de Podisus rostralis causada por duas concentrações de quatro inseticidas utilizados no controle de lagartas desfolhadoras de eucalipto.

Table 2 - Mortality (mean \pm stand error) of females and males of Podisus rostralis caused by two concentrations of four insecticides used to control eucalyptus defoliating caterpillars

\begin{tabular}{|l|c|c|c|c|}
\hline \multirow{2}{*}{ Inseticida } & \multicolumn{4}{|c|}{ Sexo* } \\
\cline { 2 - 5 } & Fêmea & Macho & Fêmea & \multicolumn{2}{c|}{ Macho } \\
\cline { 2 - 5 } & \multicolumn{2}{|c|}{ (Concentração recomendada) } & \multicolumn{2}{|c|}{ (50\% da concentração) } \\
\hline Fenitrotiom & $100,00 \pm 0,00 \mathrm{aA} \alpha$ & $100,00 \pm 0,00 \mathrm{aA} \alpha$ & $100,00 \pm 0,00 \mathrm{aA} \alpha$ & $100,00 \pm 0,00 \mathrm{aA} \alpha$ \\
Malatiom & $77,99 \pm 10,60 \mathrm{bB} \alpha$ & $100,00 \pm 0,00 \mathrm{aA} \alpha$ & $56,96 \pm 9,34 \mathrm{aB} \beta$ & $74,93 \pm 6,78 \mathrm{aB} \beta$ \\
Permetrina & $33,24 \pm 4,76 \mathrm{aC} \alpha$ & $30,92 \pm 4,89 \mathrm{aB} \alpha$ & $2,86 \pm 1,67 \mathrm{bC} \beta$ & $25,59 \pm 17,96 \mathrm{aC} \alpha$ \\
Deltametrina & $7,84 \pm 6,25 \mathrm{aD} \alpha$ & $7,89 \pm 7,89 \mathrm{aC} \alpha$ & $0,00 \pm 0,00 \mathrm{aC} \alpha$ & $1,67 \pm 1,67 \mathrm{aD} \alpha$ \\
\hline
\end{tabular}

* As médias seguidas pela mesma letra maiúscula na coluna (para comparação entre inseticidas), minúscula na linha (para comparação das diferenças de tolerância entre fêmeas e machos a uma mesma concentração de cada inseticida) ou do alfabeto grego na linha (para comparação entre concentração recomendada e $50 \%$ da concentração de cada inseticida para fêmeas e machos) não diferem entre si, pelo teste de Scott - Knott a P < 0,05.

* Means followed by same capital letter in a column (comparison between insecticides), lower case letter in a row (for comparison of the differences of tolerance between females and males to a similar concentration of insecticide) or of a Greek letter in a row (for comparison of the recommended concentration and 50\% of concentration of each insecticide to females and males) are not significantly different by the Scott-Knott test at $p<0.05$. 
predador, o qual persiste mesmo quando este predador é exposto a 50\% desta concentração. A mortalidade causada pelo malatiom para ninfas de 3 instar e adultos de ambos os sexos e por permetrina para fêmeas foi menor na subconcentração do que na concentração recomendada, já o mesmo não ocorreu como deltametrina, cuja mortalidade foi semelhante nas duas concentrações (Quadros $1 \mathrm{e}$ 2). Como o deltametrina e permetrina foram seletivos em favor do predador na concentração recomendada (Quadro 1), conclui-se que o impacto destes inseticidas é baixo no momento da aplicação. Pode-se ainda ressaltar que no caso da permetrina o efeito negativo para adultos, sobretudo para as fêmeas desse inimigo natural, é reduzido quando o predador é exposto à metade de sua concentração inicial (Quadros 1 e 2). Suinaga et al. (1996) verificaram que ocorreu redução da mortalidade causada por deltametrina e malatiom a P. nigrispinus com a exposição destes à metade das concentrações utilizadas para o controle de lagartas desfolhadoras em eucalipto, porém o mesmo não ocorreu com o fenitrotiom e o permetrina.

Com base nas taxas de degradação (Hornsby et al., 1995) dos inseticidas estudados e nos resultados obtidos, recomenda-se que em lavouras de eucalipto onde o fenitrotiom foi usado no controle de lagartas desfolhadoras o predador $P$. rostralis não deve ser liberado. Entretanto, se a pulverização for com malatiom essa liberação poderá ocorrer após a aplicação (para ninfas de 5o instar) ou dois dias após (para ninfas de 3ํ instar e adultos). Já se os inseticidas usados forem o permetrina ou o deltametrina, a liberação poderá se realizada após a aplicação, qualquer que seja o instar liberado.

\section{CONCLUSÕES}

O inseticida deltametrina é seletivo em favor de ninfas de $3^{\circ}$ e $5^{\circ}$ instares e adultos do predador P. rostralis. O permetrina é seletivo em favor de ninfas de $5^{\circ}$ instar e medianamente seletivo em favor de ninfas de $3^{\circ}$ instar e adultos do predador. O malatiom é medianamente seletivo em favor de ninfas de 5 o instar e não-seletivo em favor de ninfas de 3 instar e adultos do predador. $\mathrm{O}$ fenitrotiom não é seletivo a nenhum dos instares do predador.

Mesmo com a exposição de $P$. rostralis à metade da concentração do fenitrotiom, este inseticida ainda apresenta alta toxicidade ao predador. O impacto do malatiom a ninfas de 3 instar e adultos e do permetrina a fêmeas de $P$. rostralis diminui quando estas fases são expostas à metade das concentrações destes inseticidas, no entanto tal variação não é verificada para o deltametrina.

As ninfas de 3o instar e adultos de $P$. rostralis são menos tolerantes que as ninfas de 5- instar ao malatiom e permetrina. As fêmeas de $P$. rostralis são mais tolerantes que machos à concentração de malatiom e subconcentração de permetrina.

\section{AGRADECIMENTO}

Ao Mestre em Entomologia Eliseu José Guedes Pereira, pela revisão do manuscrito, e à CAPES (Coordenação de Aperfeiçoamento de Pessoal de Nível Superior) e ao CNPq (Conselho Nacional de Desenvolvimento Científico e Tecnológico), pelas bolsas concedidas.

\section{REFERÊNCIAS BIBLIOGRÁFICAS}

ABBOTT, W. S. A method of computing the effectiveness of an insecticide. Journal of Economic Entomology, v. 18, n. 1, p. 265-267, 1925.

ANDREI, E. Compêndio de defensivos agrícolas. 5.ed. São Paulo: Andrei, 1996. 506 p.

BATALHA, V. C. et al. Seletividade de inseticidas aos predadores Podisus nigrispinus (Dallas, 1851) e Supputius cincticeps (Stal, 1860) (Heteroptera: Pentatomidae) e a sua presa Lepidoptera. Revista Árvore, v. 19, n. 3, p. 382-395, 1995.

EUCLIDES, R. F. Sistema para análises estatísticas e genéticas. Viçosa-MG: UFV, 1983. 37 p.

FALEIRO, F. G. et al. Seletividade de inseticidas a Spodoptera frugiperda (J.E. Smith) (Lepidoptera: Noctuidae) e o predador Doru luteipes (Scudder) (Dermaptera: Forficulidae). Anais da Sociedade Entomológica do Brasil, v. 24, n. 2, p. 247-252, 1995.

GONRING, A. H. R. et al. Seletividade de inseticidas, utilizados no controle de Grapholita molesta (Busch) (Lepidoptera: Olethreutidae) em pêssego, a Vespidae predadores. Anais da Sociedade Entomológica do Brasil, v. 28, n. 2, p. 301-306, 1999.

GUEDES, R. N. C.; LIMA, J. O. G.; ZANUNCIO, J. C. Seletividade dos inseticidas deltametrina, fenvalerato e fenitrotiom para Podisus connexivus (Heteroptera: Pentatomidae). Anais da Sociedade Entomológica do Brasil, v. 21, n. 3, p. 339-346, 1992.

R. Árvore, Viçosa-MG, v.27, n.2, p.263-268, 2003 
HOLLINGWORTH, R. M. The biochemical and physiological basis of selective toxicity. In: WILKINSON, C. F. (Ed.), Insecticide Biochemistry and Physiology. New York, Plenum, 1976. p. 431-506.

HORNSBY, A. G.; DON WAUCHOPE, R.; HERNER, A. E. Pesticide properties in the environment. New York: Springer, 1995. 227 p.

HOY, M. A. Pesticide resistance in arthropod natural enemies: variability and selection response. In: ROUSH, R.T., TABASHNIK, E. (Eds.). Pesticide resistance in arthropods. New York, Chapman and Hall, 1990. p. 203236.

LEGASPI, J. C.; O’NEIL, R. J.; LEGASPI JR., B. C. Trade-offs in body weights, egg loads, and fat reserves of field-collected Podisus maculiventris (Heteroptera: Pentatomidae). Environmental Entomology, v. 25, n. 1, p. $155-164,1996$.

PEDIGO, L. P. Entomology and pest management. New York: Macmillan. 1988. 646 p.

PICANÇO, M. et al. Seletividade de inseticidas a Polybia ignobilis (Haliday) (Hymenoptera: Vespidae) predador de Ascia monuste orseis (Godart) (Lepidoptera: Pieridae). Anais da Sociedade Entomológica do Brasil, v. 27, n. 1, p. 73-78, 1998.

PICANÇO, M. et al. Seletividade de inseticidas a Podisus nigrispinus predador de Ascia monuste orseis. Pesquisa Agropecuária Brasileira, v. 32, n. 4, p. 369-372, 1997.

POWELL, J. E.; KING JR., E. G.; JANY, C. S. Toxicity of insecticides to adult Microplitis croceipes (Hymenoptera: Braconidae). Journal of Economic Entomology, v. 79, n.5, p. 1343-1346, 1986.

RAJAKULENDRAN, S.V.; PLAPP JR., F. W. Comparative toxicities of five synthetic pyrethroides to the tobacco budworm (Lepidopetra: Noctuidae), an Ichneumonidae parasite, Campoletis sonorensis, and a predator, Chrysopa carnea. Journal of Economic Entomology, v. 78, n. 1, p. $769-772,1982$.
SCOTT, A. J.; KNOTT, M. A. A cluster analysis method for grouping means in the analysis of variance. Biometrics, v. 30, n. 3 , p. 507-512, 1974.

SINGH, P. P.; GUARDIAL, C. V. Comparative toxicities of some insecticides to Chrysoperla carnea (Neuroptera: Chrysopidae) and Trichogramma brasiliensis (Hymenoptera: Trichogrammatidae), two arthropod natural enemies of cotton pests. Agriculture, Ecosystems \& Environment, v. 15, n. 1, p. 23-30, 1986.

SUINAGA, F. A. et al. Seletividade fisiológica de inseticidas a Podisus nigrispinus (Dallas, 1851) (Heteroptera: Pentatomidae) predador de lagartas desfolhadoras de eucalipto. Revista Árvore, v. 20, n. 3, p. 407-414, 1996.

WINTERINGHAM, F. P. W. Mechanisms of selective insecticidal action. Annual Review Entomology, v. 14, p. 409-442, 1969

YU, S. J. Selectivity of insecticides to the spined soldier bug (Heteroptera: Pentatomidae) and its lepidopterous prey. Journal of Economic Entomology, v. 81, n. 1, p. 119-122, 1988.

ZANUNCIO, J. C.; ALVES, J. B.; ZANUNCIO, T. V. VI Hemípteros predadores de lagartas desfolhadoras de eucalipto. In: ZANUNCIO, J. C. (Ed.). Lepidoptera desfolhadores de eucalipto: Biologia, ecologia e controle. Viçosa-MG: IPEF/SIF, 1993a. p. 107-125.

ZANUNCIO, J. C. et al. Métodos para criação de hemípteros predadores de lagartas. Anais da Sociedade Entomológica do Brasil, v. 21, n. 2, p. 245-251, 1992a.

ZANUNCIO, J. C. et al. Eficiência de Bacillus thuringiensis e de deltametrina, em aplicação aérea, para o controle de Thyrinteina arnobia Stoll, 1782 (Lepidoptera: Geometridae) em eucaliptal no Pará. Acta Amazônia, v. 22, n. 4, p. 485-492, 1992 b.

ZANUNCIO, J. C. et al. Impact of two formulations of deltamethrin in aerial application against Eucalyptus caterpillars and their predaceous bugs. Medical Faculteit Landbouwkundige, v. 58, n. 2a, p. 469-475, 1993b. 